\title{
Ciudad, arquitecturas y restauraciones, problemas de conservación de los cascos históricos
}

\author{
City, architecture and restoration, conservation \\ problems of historical centers
}

G. Minutoli $^{(*)}$

RESUMEN

Abre el artículo un breve recorrido histórico sobre las normas para la restauración, dando las principales referencias hasta llegar al concepto de protección del "moderno". Cierra esta primera parte del artículo, una breve anotación sobre los trabajos Ilevados a cabo en Florencia capital, durante el siglo diecinueve, intervenciones que han influido considerablemente en la moderna practica de restauración.

En la parte central del artículo, se estudia la metodología considerada más apropiada para la intervención en los centros históricos, con proyectos de restauración urbana que no se limitan a mejorar el tejido urbano en su imagen. Sino en otros aspectos como los estructurales y funcionales, dirigidos a conseguir una mayor habitabilidad y disfrute de las ciudades históricas, con ejemplos de intervenciones más o menos logradas. Dos ejemplos prácticos de intervenciones cierran el artículo, la restauración del Palazzo Dami en Florencia y la restauración y consolidación de algunas zonas del Palazzo Uffizi en la misma ciudad.

Palabras clave: Metodología; intervención en centros históricos; tejido urbano; aspectos estructurales; aspectos funcionales.

\section{SUMMARY}

This writing begins revisiting the theories which gave the principle references for the restoration of monuments, till the protection of the "modern". The first part of this article finished with a light description of the restoration works that took place in Firenze at the end of the nineteen century.

Afterwards, it tries to explain the methods used in the restoration of historic towns, attending to other subjects, not only to image. But to structural and functional meanings, in order to be able to improve the way of living in the historic towns.

Practical examples of restoration interventions as those of Palazzo Dami and Palazzo Uffizi in Firenze, close this article.

Keywords: Method; restoration of historic towns; urban context; structural meanings; functional meanings.

(*) Università degli Studi di Firenze, Florencia (Italia). 


\section{UN BREVE RECORRIDO POR EL ESTADO DE LA CUESTIÓN}

El problema de la conservación del patrimonio histórico, es desde siempre objeto de debates, no solo entre los arquitectos, sino también entre los intelectuales de todas las civilizaciones; Plutarco ${ }^{1}$ da a conocer como en Atenas, los filósofos se preguntaban sobre la "autenticidad" del buque de Teseo. El buque, conservado durante siglos, requería continuas sustituciones de las partes deterioradas, hasta llegar a que nada de lo conservado perteneciera a su materia original. Los filósofos se preguntaban, si el buque conservado con tanto cuidado durante siglos, debía considerarse el de Teseo. Una facción apoyaba la idea de que no conservándose elementos originales, no se podría identificar más aquel buque con el de Teseo; mientras que otro grupo defendía el hecho de que habiendo mantenido la imagen antigua con las sustituciones de materia, el buque debía considerarse original.

Haciendo un recorrido a través de los siglos, en edad clásica romana, el concepto de restauración se confunde con el de mantenimiento, pero está vinculado más al mundo de las obras de arte (bienes muebles) que a la arquitectura. Vitruvio, Plinio y Pausanias en sus escritos, explican qué argucias empleaban los artistas del mundo clásico para el mantenimiento de sus obras. Se empieza a hablar de conservación de los edificios con el emperador Teodosio, que en numerosos edictos impone la protección de los edificios públicos, y prohíbe el expolio de obras artísticas, reservando para su conservación un tercio de los ingresos del erario (1).

Con Teodosio II y Valentiniano II, en el siglo IV después de Cristo, se autoriza la reutilización de los antiguos templos y su reconversión en iglesias cristianas (1); costumbre que se mantendrá durante toda la Edad Media y que verá también intervenciones excepcionales como la de Miguel Ángel en las termas de Diocleziano, transformadas en basílica de Santa María de los Ángeles. El uso de edificios de época clásica, como el teatro de Marcello en Roma, el anfiteatro romano de Florencia, el templo de Atenea en Siracusa, sólo por mencionar algunos ejemplos, pone el acento sobre la reutilización más que sobre la restauración. La finalidad de las intervenciones realizadas durante la Edad Media y el Renacimiento no fue la de conservar el elemento arquitectónico para poder transmitirlo a la posteridad, sino que fue meramente funcional, teniendo como punto principal la reutilización.
En la misma época, arqueólogos, historiadores y arquitectos descubren y estudian los vestigios romanos, siendo en este período cuando numerosas obras de arte clásicas entran a formar parte de colecciones privadas. Los arquitectos estudian y dibujan las ruinas romanas, pasando los modelos y los elementos arquitectónicos clásicos, a formar parte del repertorio fundamental de la arquitectura renacentista y manierista.

Y será en el setecientos, cuando se desarrolle la conciencia conservadora de la que todavía hoy estamos influidos, cuando Antonie Crysostome Quatremère de Quincy publica las Cartas a Miranda. En el texto, el autor invita a los arquitectos a conservar los monumentos en su integridad histórica y en sus contextos culturales.

Para encontrar las primeras normas relativas a la protección del patrimonio históricoartístico, habrá que esperar al siglo diecinueve, cuando se promulgó en el Estado Pontificio el Edicto Pacca. Éste, prescribía el inventario de las obras de arte, tanto públicas como privadas, y prohibía la exportación y la enajenación de cualquier objeto de valor histórico-artístico procedente de iglesias, monasterios, etc. El texto, completado el 6 agosto 1821, extenderá la protección a los objetos de arte considerados menores.

Esta ley se hizo necesaria por el creciente interés hacia las obras de arte italianas, debido al nacimiento de numerosos museos en todo el mundo que requerían objetos de arte originarias de Italia (2).

Un precedente de catalogación, se encontrará en la legislación cisalpina, más precisamente en la circular del Ministerio del Interior del 13 julio 1798, en la que se requería la elaboración de un inventario de los objetos artísticos procedentes de iglesias, archivos, bibliotecas etc. Entre ellos, también de los manuscritos, medallas, marcos etc, debiendo entregar una copia a las autoridades competentes en la materia y dejando los originales de las mismas, en custodia provisional, a los poseedores. En la norma se especificaba no sólo el valor histórico-artístico del bien, sino también su valor didáctico, necesario para el estudio de las nuevas generaciones.

Paralelamente iba desarrollándose el concepto de restauración, que primitivamente fue vinculado al redescubrimiento de los monumentos medievales, probablemente porque en los primeros decenios del siglo XIX, el interés por la Edad Media y el Renacimiento por parte de los historiadores había influido también en los arquitectos. No se había aún determinado el significado de la
1 Plutarco. Vite Parallele. Vite di Romolo e di Teseo,23.I 
palabra "restauración", que en este período era confusa en su aspecto operativo o practico, ligado más bien a la "reconstrucción". Es fácil encontrar en este período, edificios de planta gótica reconstruidos en estilo neogótico, porque considerados vetustos o degradados, fueron completados en estilos medievales extraídos de los tratados contemporáneos; siendo difícil distinguir entre los edificios nuevos y los reconstruidos y/o completados pero de antiguo origen. Hay que aclarar que las intervenciones de reconstrucción "en estilo", no eran consideradas obras de restauración en el ámbito italiano por autores como Camillo Boito. Ya en el año 1883 en ámbito internacional, y alrededor de un siglo antes, por Quatremère de Quince, estos conceptos fueron difundidos mediante circulares y cartas de restauración (2).

La cultura de la restauración, en estos primeros decenios del siglo XIX, se enriquecerá con las intervenciones en el Arco de Tito y en el Coliseo de Roma; ambas intervenciones pasarán a ser puntos de referencia en la naciente cultura de la restauración. Este concepto de conservación, con el transcurso del tiempo se ampliará, pasando de la protección de los objetos a la de los edificios...y a la protección de sectores urbanos completos.

Al mismo tiempo, diferentes ciudades europeas, como Londres y París, serán objeto de importantes intervenciones de renovación urbana, con actuaciones que modificarán radicalmente su imagen.

\section{FLORENCIA}

En el ámbito italiano, con la elección de Florencia como capital, se plantearon numerosas preguntas al respecto. Entre ellas, la de cómo intervenir en una amplia zona central y en profundo estado de deterioro, como la comprendida entre la plaza de la Catedral, Via Calzaioli, el Mercado nuevo y el Palacio Strozzi, el gueto.

El primer proyecto de intervención en la parte central del gueto es del año 1865, cuando el ingeniero municipal Luigi del Sarto, basándose en las experiencias de otras ciudades europeas, propuso un intervención que iría desde la realización de una galería cubierta por los cuatro lados, de la plaza del mercado viejo (Figuras 1 y 2 ) a la lonja de Pesce. El proyecto aprobado en el 1866 por el Consejo Municipal, Ilevó consigo la más amplia obra de demolición y reconstrucción que jamás haya sufrido el centro histórico de Florencia. Los trabajos continuaron con extrema lentitud, tanto que en 1881 el proyecto de del Sarto, aún no realizado, fue revisado y modificado por de Fabbris, antes de la reanudación de las obras.
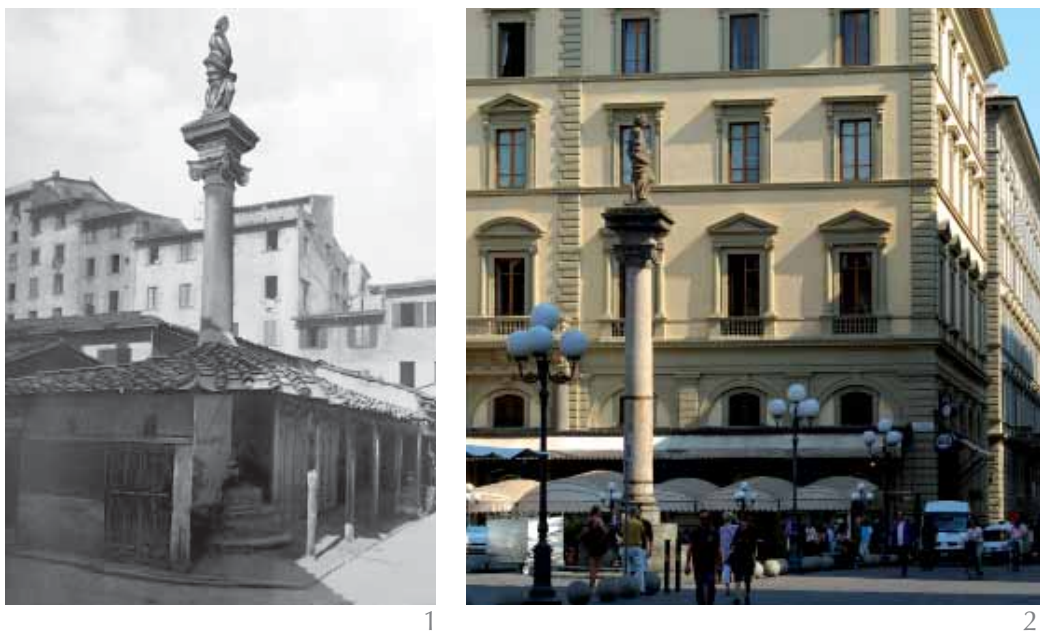

Se opusieron a esta propuesta de demoliciones parciales, los Colegios de ingenieros-arquitectos, que querían la total demolición del "tejido menor", proponiendo salvar únicamente los edificios singulares ("de mérito"). El debate sobre el valor de esta zona del centro urbano, y sobre cuánto y qué demoler, duró varios años, hasta que en 1885 una epidemia de cólera afectó al centro histórico de Nápoles, convenciendo así a los habitantes de Florencia de la necesidad de intervenir en su centro histórico.

Los trabajos comenzaron efectivamente en 1885, y fueron seguidos por una subcomisión municipal, encabezada por Luigi del Moro, Guido Carocci y Gaetano Milanesi, que se oponían a las grandes demoliciones; la documentación relativa a éstas y a las excavaciones, fue garantizada por los levantamientos de Corinto Corinti y las fotografías del estudio Brogi. La intervención se amplió con la demolición de una parte de las murallas, y la realización del viale dei Colli; las obras se concluyeron en 1892, con la colocación de la lápida conmemorativa sobre el arco que hoy se abre entre la plaza de la República y la calle Strozzi

La imagen de buena parte de la ciudad de los Medici cambió irreversiblemente; de medieval y caótica, pasó a convertirse en ochocentista y ordenada. Sólo las imágenes de los Brogi y la documentación detaIlada realizada por Corinto Corinti (Figura 3), nos permite imaginar cómo era la ciudad, con qué estaba apareciendo durante las excavaciones y cual sería la nueva imagen de los lugares objeto de intervención. Una serie de tarjetas ilustradas por el mismo Corinti documentan las fases relevantes de las excavaciones. Algunos elementos interesantes, fueron recogidos en los círculos del naciente Museo Arqueológico Nacional, donde fueron expuestos de forma filológica mientras otros se conservaban al aire libre.
1. Florencia, Piazza del Mercato Vecchio, antes de la demolición.

2. Florencia, Piazza della Repubblica en la actualidad, antigua Piazza del Mercato Vecchio. 
3. Excavación en Via di Calimara. (Ilustración de Corinto Corinti).

4. Florencia, Via Borgo Santissimi Apostoli, su recorrido sigue siendo medieval.

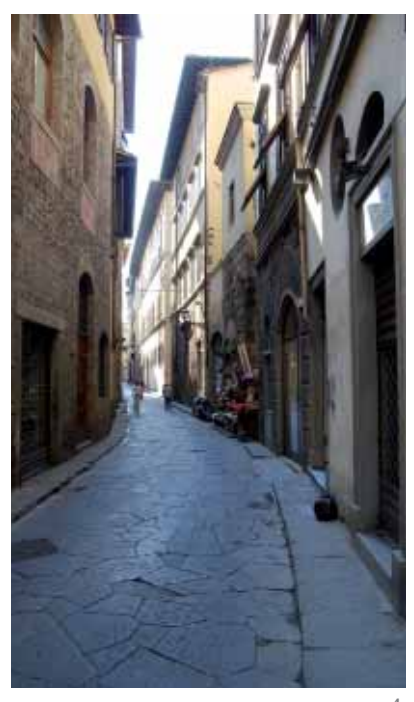

${ }^{2}$ Se han citado únicamente los principales textos de leyes que han servido de base a la normativa sucesiva.

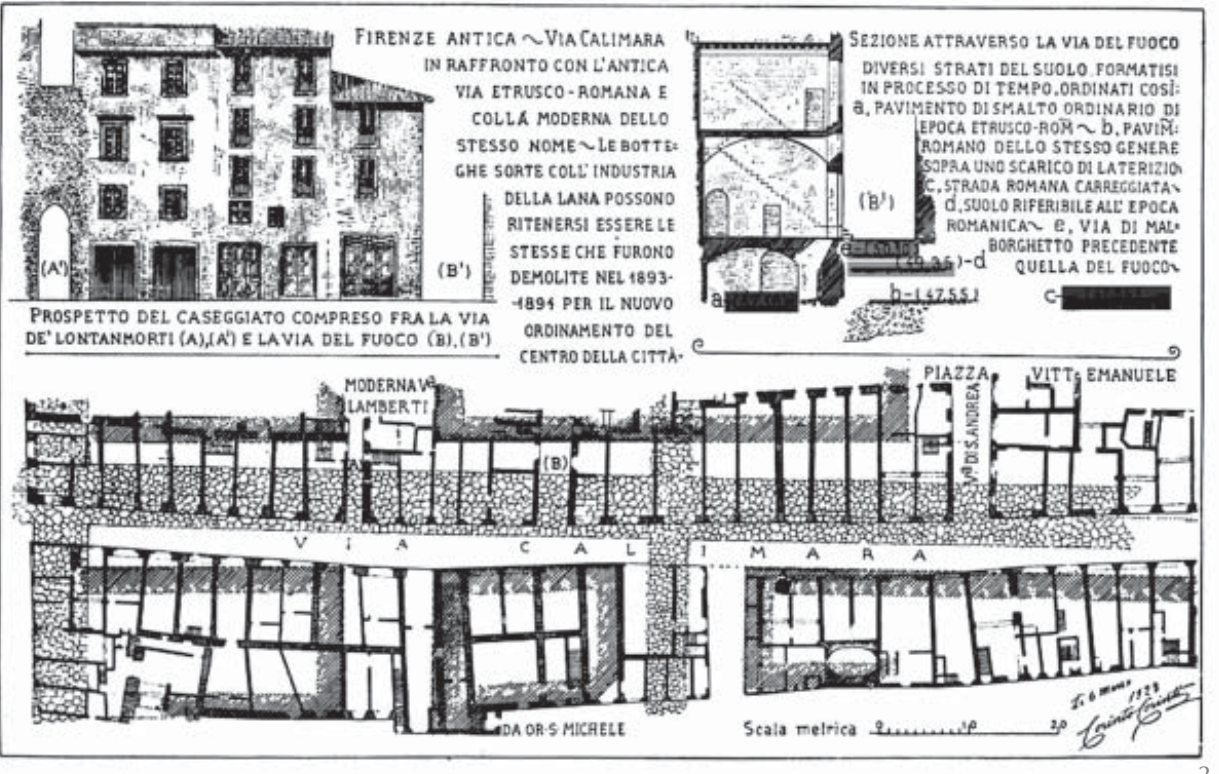

A partir de entonces, quien visite Florencia tendrá la idea de una ciudad del siglo diecinueve, con una amalgama de monumentos medievales junto a otros del renacimiento. Sólo en unos pocos ámbitos es aún detectable el carácter medieval de la ciudad; como la zona comprendida entre Via di Porta Rossa y el Lungarno Acciaioli, con sus vías tortuosas, y callejones estrechos (Figura 4).

En los mismos años en que se discutía, en un marco nacional, sobre el destino del centro histórico de Florencia, también empezaban a surgir las preguntas sobre cómo salvaguardar el cuantioso patrimonio de la Italia unida.

En el 1875 se decidió redactar "la lista de los Monumentos Nacionales Medievales y Modernos"; en 1883 este listado se ampliará y profundizará. La catalogación del patrimonio arquitectónico nacional, sirvió como base para la elaboración de la Ley 185 de 1902 sobre la conservación de los "objetos" de arte y antigüedad y por primera vez se limita el derecho de los privados sobre sus bienes.

Figuras destacadas en el ámbito de la restauración, durante todo el último cuarto del siglo XIX y el primer decenio del siglo XX serán Camillo Boito, y Gustavo Giovannoni, fuertemente influenciado por aquel. Éste, en la primera mitad del siglo XX, dirigirá la actividad de los "restauradores" italianos, aunque no logrando contener ni los excesos de algunos arquitectos en la búsqueda de la "fábrica originaria", ni las exageradas demoliciones del régimen fascista.

Giovannoni, en el ámbito académico, colaborará en la Facultad de Arquitectura, en la creación de los cursos de Historia de la Arquitectura y de tipologías arquitectónicas, completando así el bagaje cultural de los arquitectos italianos, y permitiendo a dichos técnicos profundizar en algunos aspectos históricos, que hasta ese momento eran monopolio de los historiadores del arte.

En 1939 se aprueban por la Cámara Italiana, dos textos fundamentales para la salvaguarda del rico patrimonio artístico y natural italiano: la Ley 1089 para la Protección del Patrimonio Histórico-Artístico y la Ley 1497 para la Protección de las Bellezas Naturales y Características del Territorio².

La extensa correspondencia entre Giovannoni y el Ministro de Educación Nacional Giuseppe Bottai, muestra cuanto pudo haber influido Giovannoni en el mismo para la elaboración de ambas leyes (3). Estos dos textos, son vistos por la opinión pública como una limitación de la libertad personal, en cuanto someten el interés privado al público. Por su gran modernidad, pasan a ser punto de referencia cultural de muchos países europeos, influyendo en la normativa de muchas naciones. Ampliaron el concepto de protección del elemento aislado, a una concepción global del patrimonio, abarcando tanto aspectos urbanísticos como paisajísticos.

Al mismo tiempo evolucionó también en el concepto de restauración, que se fue ampliando hacia el paisaje, siguiendo así las indicaciones de la Ley 1497 de 1939,citada anteriormente, filosofía posteriormente ampliada por Roberto Pane y reiterada en 1978 por Francesco Gurrieri en el texto titulado: "Dal restauro dei monumento al restauro del paesaggio". Más tarde también se amplió, hacia la restauración de edificios construidos en la primera mitad del siglo $X X$, que después de cincuenta años de vida comienzan a requerir las primeras intervenciones de adecuación y de restauración. 
En el primer período de posguerra, las restauraciones sobre las arquitecturas del siglo XX se ocuparon casi exclusivamente de los edificios de los años veinte, en estilo "racionalista europeo", excluyendo de hecho aquellos edificios considerados "monumentales". Posteriormente, también estos últimos comienzan a ser objeto de intervenciones rigurosas y científicas, dirigidas a la salvaguarda tanto de la estabilidad física del edificio como de su imagen.

Con los bombardeos de la segunda guerra mundial, Florencia sufre daños en su tejido urbano. Las bombas afectan tanto a los que se considerarán "verdaderos monumentos", como el puente de Santa Trinidad, como también a buena parte del tejido menor, que cierra al Sur y al Norte el Ponte Vecchio. En efecto, los alemanes para no bombardear el puente más antiguo de la ciudad, destruyeron sus cabeceras, entre las Vías Santa María, Gucciardini y Borgo San Jacopo. Estos tres sectores urbanos se reconstruyeron en la posguerra, buscando así dotar de sentido moderno a aquella porción de la ciudad.

Si se tiene en cuenta el centro histórico de Florencia, la idea preconcebida que el turista tiene al recorrerlo, es la de una ciudad medieval y renacentista; pero en realidad, los ejes principales frecuentados por los visitantes, es decir Vía Calzaioli y Via Strozzi, como ya se ha dicho, son el fruto de la importante intervención del siglo diecinueve, donde la ciudad fue readaptada a las necesidades y al gusto de esa época, a la búsqueda de una idea de pureza del Renacimiento que nunca ha existido.
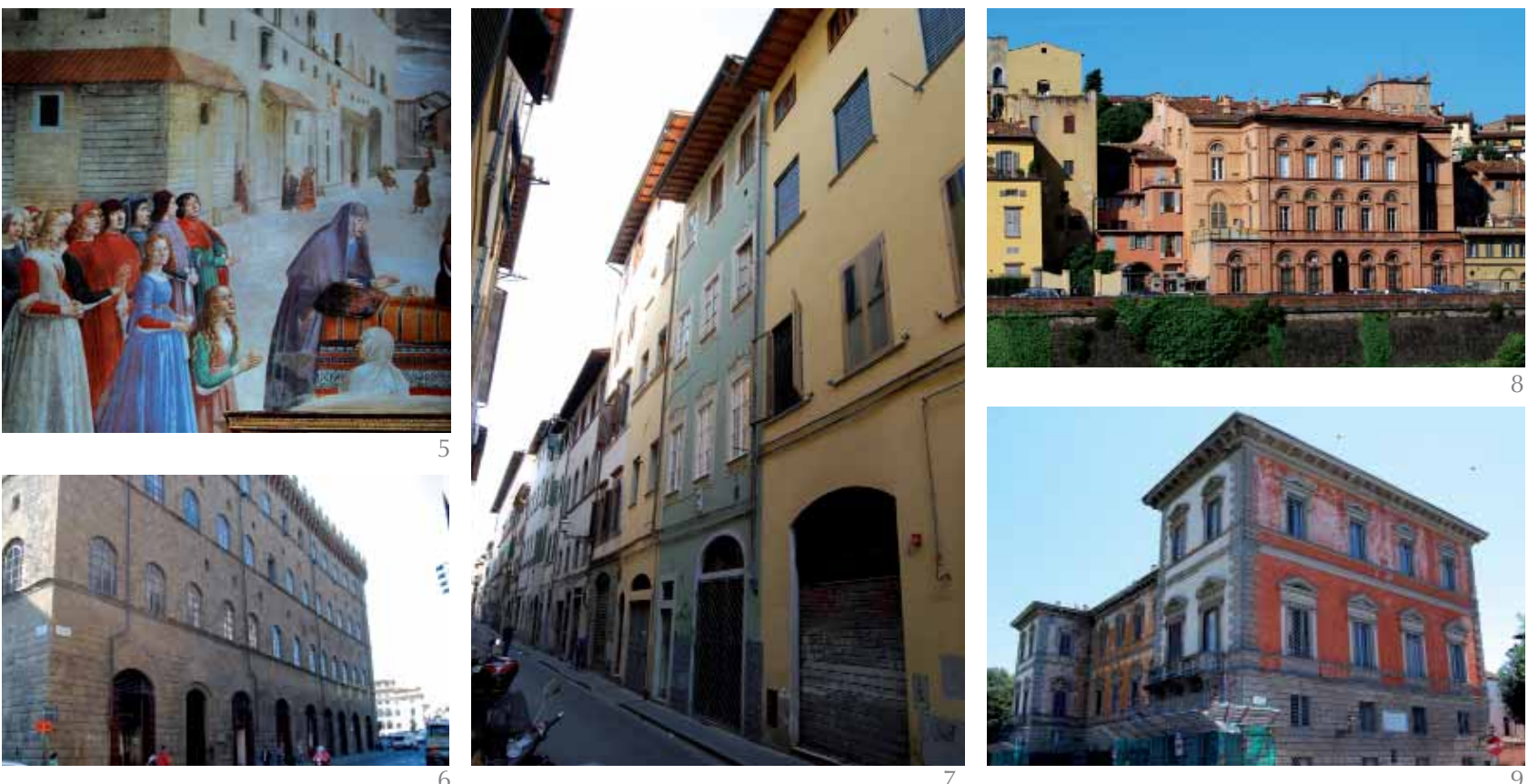

En esos años, la ciudad no considerada como un conjunto, es decir un organismo todo él monumental, sino como una suma de monumentos aislados: el Duomo, el Palacio Viejo, el Palacio Strozzi, etc., como si estos para existir no necesitasen del "tejido menor".

La producción pictórica del trecento y del cuatrocento documenta la ciudad de la época, con fachadas desordenadas y carentes de simetría, calles con suelo de tierra y con ejes no rectilíneos de origen medieval, muy alejada de la imagen de la "ciudad ideal" tan próxima a los estudiosos del renacimiento.

En el luneto central del fresco del Ghirlandaio, en la Capilla Sassetti en Santa Trinitá, se ve una representación de la plaza que da frente al citado templo, fechada en el cuatrocento. El palacio Spini Feroni, en primer plano, resulta muy distinto a su imagen actual., con un aspecto no homogéneo de estilo medieval (Figuras 5 y 6). Otro ejemplo significativo se encuentra en la capilla Brancacci de la iglesia Santa María del Carmine, donde fragmentos de arquitecturas del cuatrocento sirven de trasfondo a las escenas bíblicas.

En realidad, en el centro histórico de Florencia se pueden encontrar aún algunos fragmentos de ciudad antigua, que conservan los rasgos característicos del renacimiento: Via Borgo SS. Apóstoles, Via delle Terme, Via San Niccolò, vias que no han sufrido grandes obras de renovación urbana, y cuyos edificios conservan en sus fachadas una tenue coloración, propia del ochocientos.
5. Florencia, Iglesia de Santa Trinita, capilla Sassetti, Ghirlandaio, detalle de la pintura que forma parte del Palazzo Spini Ferroni de mitad del Siglo XV.

6. Florencia, Palazzo Spini Ferroni en la actualidad.

7. Florencia, Via Borgo Tegolaio, fachada rococó del edificio con sus colores originales.

8. Florencia, Lungarno Torrigiani, Palazzo Capponi delle Rovinate, su combinación de colores es el original.

9. Florencia, Lungarno Serristori Palace Serristori con su variedad de colores, indicativos de las diversas fases de restauración. 
Dispersos por el centro histórico, se encuentran también ejemplos de edificios en los que recientes restauraciones han puesto en evidencia los colores originales de las fachadas; se citan como ejemplos significativos, un edificio en estilo Rococó en Borgo Tegolaio con acabado verde-azul (Figura 7), el Palazzo Capponi delle Rovinate en Lungarno Torrigiani, color rojo ladrillo (Figura 8), el palazzo Serristori en el mismo callejón, con algunas fachadas rojas y otras blancas y grises, que ponen de manifiesto intervenciones correspondientes a diferentes épocas (Figura 9).Estos y otros pocos edificios se distinguen del resto del conjunto urbano con sus tenues coloraciones, entre el gris y el blanco, propios del siglo XIX.

\section{PRESENTE Y FUTURO}

Hace unos veinte años que se llevó a cabo la peatonalización de todo el centro histórico de la ciudad de los Medicis; el primer tramo que se cerró al tráfico, el comprendido entre la Piazza del Duomo y la Piazza della Signoria; más tarde fue cerrado al tránsito rodado todo el interior de la ciudad, que está comprendido dentro del perímetro de la ciudad romana.

El alcalde Matteo Renzi, desde el año 2009 ha reactivado el cierre del centro al tráfico rodado, ampliando la zona peatonal hasta conectar la Piazza del Duomo con el Palazzo Medici Riccardi por el Norte, y el Ponte Vecchio con el Palazzo Pitti, al Sur. Al mismo tiempo, se comenzó la reparación de la pavimentación de algunas vías del centro histórico.

Cualquier elección de proyecto, se hace cuestionable si no está respaldada por una idea general de ciudad, que la considere en todos sus aspectos, y entre aquellos a decidir será el relativo a sus tonalidades de color. Muchos centros de investigación, tanto públicos como privados, han concentrado sus energías en la búsqueda de la coloración histórica de edificios de muchas ciudades italianas. Como consecuencia se han realizado cartas de color para la restauración de fachadas, según su localización geográficas.

En cuanto a la técnica, el uso de materiales acrílicos, ha conducido a realizaciones incompatibles con el organismo edificatorio al que pertenecen; en la mayoría de los casos el uso de revocos preparados, aplicados en forma industrial, ha hecho virar los colores originales, transformando el aspecto de la fachada.

Piero Roselli (4), señala como objetivo para la restauración urbana "el de conservar los caracteres arquitectónicos, espaciales y medioambientales que están en un determinado centro, y que han conferido al mismo una precisa connotación y una fisonomía que lo diferencia de cualquier otro centro, teniendo sin embargo en cuenta que no está constituido exclusivamente por monumentos y que no se puede y no se debe hacer llegar a ser monumento cada elemento que constituye la estructura urbana".

Es evidente que no hay que impedir, con normas especialmente restrictivas, la adaptación del tejido urbano y de las arquitecturas que lo componen, a las variables necesidades de uso; resultaría negativo para el desarrollo de cualquier centro urbano que el plan de recuperación propusiera como instrumentos la congelación de lo existente.

El objetivo fundamental de cualquier "plan de recualificación urbana", será convertirse en medio para adaptar los tejidos históricos a las necesidades de la vida contemporánea, estableciendo con el pasado un vínculo, como signo de continuidad. Será fundamental entender que los planes de recuperación urbana deben ocuparse de otros aspectos, no únicamente de operaciones de make-up, como el "plan del color", que limita su acción exclusivamente al aspecto cromático y/o a proyectos de mobiliario urbano.

Para el éxito de un plan de recuperación urbana, será necesario que participen los entes públicos y que hagan compartir las elecciones de proyecto con la iniciativa privada; sin la intervención combinada de ambos, no será posible recuperar las distintas formaciones urbanas. La creación de una buena red de infraestructuras urbanas y el diseño de los espacios públicos no bastarán para la mejora del medio ambiente urbano, así como la restauración arquitectónica no equivaldrá a un plan de recuperación.

La recuperación de la escala urbana de un centro histórico, por grande o pequeño que sea, debería contemplar tres fases de análisis previos al proyecto, $y$ tres fases de intervención en la actuación.

Consideramos fases de análisis previo:

1. Investigación histórico-documental sobre el casco urbano y sobre las arquitecturas que lo componen.

2.Levantamiento a escala del centro histórico y de las arquitecturas más significativas.

3. Estudio de las técnicas constructivas y de los elementos característicos y elaboración de fichas con riesgos tales como los de vulnerabilidad sísmica.

Y considerando las fases de actuación:

1.Reordenación y mantenimiento de las infraestructuras. 
2.Consolidación del sector espacial en el que se desarrolla el centro histórico y las distintas unidades que lo componen.

3.Restauración de las fachadas y recuperación de su diseño.

En la primera de las tres fases de análisis, la elaboración del estudio histórico a escala permitirá localizar todas las informaciones necesarias para la comprensión del desarrollo urbanístico de la ciudad, y de los distintos conjuntos arquitectónicos característicos del núcleo urbano. La historia sísmica, completará la información fundamental para la comprensión de las fragilidades del lugar. La investigación histórica no debería limitarse al simple inventariado de fuentes documentales, sino que será necesario el examen de los archivos públicos y privados, en la búsqueda de las informaciones que los historiógrafos hubieran omitido o mal interpretado, según la metodología actual.

La segunda fase prevé el levantamiento arquitectónico, el análisis de los sistemas constructivos y de sus materiales así como del deterioro de los edificios, incluido el de los pavimentos viarios y el de las infraestructuras urbanas. El levantamiento pondrá en evidencia todos esos "signos" característicos de los distintos centros históricos, ayudando al proyectista a comprender el lenguaje arquitectónico local y sus mutaciones a lo largo del tiempo.

La tercera fase prevé el estudio de las técnicas constructivas, así como de los elementos característicos de la arquitectura y la elaboración de las fichas de vulnerabilidad sísmica. El estudio de las técnicas constructivas y de los elementos decorativos no debe ser hecho por áreas geográficas extensas, puesto que cada centro urbano tiene sus materiales y su tradición constructiva.

Por lo general las arquitecturas y sus elementos decorativos, se han venido realizando según técnicas y estilos locales, utilizados por constructores y artesanos del lugar, aunque a veces estos mostrasen referencias de ámbito regional. Para la evaluación de la vulnerabilidad sísmica u otros riesgos, será necesario que para cada centro urbano, se modifique o se amplié el modelo base de la ficha de vulnerabilidad, teniendo presentes las particularidades urbanas, arquitectónicas y constructivas de cada centro.

Será necesario, que en la redacción de las fichas de vulnerabilidad, se inscriban los factores de fiabilidad y los niveles de conocimiento, según lo solicitado en materia de construcción de fábricas.
La fase de análisis será fundamental para el proyectista, en cuanto pondrá en evidencia los que serán puntos clave del proyecto de recuperación, aunque dejando al diseñador los márgenes de interpretación personal. La primera fase de intervención se refiere a la adaptación de las infraestructuras preexistentes: redes de saneamiento, abastecimiento de agua potable y redes tecnológicas. Antes de pasar a las intervenciones más evidentes, será necesario prever el mantenimiento extraordinario de todas las instalaciones existentes bajo el pavimento viario, proyectando también el soterramiento de redes aéreas existentes, como la eléctrica y la telefónica, permitiendo así eliminar de las fachadas los cableados etc, que constituyen una evidente forma de degradación de nuestros centros históricos.

Es de subrayar como las nuevas redes que sustituyan a las existentes, casi siempre obsoletas, y los planes de mantenimiento que les afecten, tienden con frecuencia a solucionar emergencias, pero sin un preciso plan de intervenciones orientadas a la modernización. Si para las instalaciones de abastecimiento de agua potable y de alcantarillado, por norma se ha debido elaborar un proyecto preventivo, para la realización y la ampliación de redes tecnológicas se ha venido actuando sólo dando satisfacción a las cambiantes necesidades, sin prever un anteproyecto unitario y sin preocuparse de la eliminación de las líneas en desuso o de las instalaciones anticuadas.

La segunda fase de intervención, quizá la más importante tanto desde el punto de vista económico como social, y también la más compleja de administrar, prevé la intervención combinada entre lo público y lo privado. En efecto, antes de proceder a dar respuestas formales, como el cuidado de fachadas y del mobiliario urbano, será necesario dar respuesta a aspectos estructurales que garanticen la estabilidad de lo existente, incluso de seguridad frente al sismo donde sea necesario. Sobre la base de los datos suministrados por la elaboración de las fichas de vulnerabilidad sísmica y el estudio de las técnicas constructivas locales, será posible individualizar las fragilidad de cada edificio, dando así al proyectista todas las indicaciones necesarias para intervenir en los edificios existentes, con proyectos de consolidación que no sólo conserven el edificio singular, sino también su entorno, los inmuebles que lo rodean.

La tercera fase, que prevé la recuperación de la imagen del centro histórico, tendrá como punto fundamental la conservación y el desarrollo de la percepción de la ciudad y de sus componentes en los espacios públicos, 
10. Florencia, Via dell'Agnolo, conjunto de las Murate.

11. Florencia, conjunto de lasMurate, plaza central.

12. Florencia, conjunto de las Murate, pasarela cubierta que COnecta las dos calles principales. y debería observar una vez más, la relación entre las intervenciones de ámbito público y privado. Resueltas las cuestiones relativas a las instalaciones y la consolidación de los edificios, se procederá con aquellas intervenciones dirigidas a recalificar y modernizar el aspecto visual del Centro Histórico. Las primeras intervenciones se dirigirán a actuar sobre las fachadas de los edificios más antiguos, con la restauración de revestimientos, revocos y su color, con la eliminación de elementos tales como antenas..., que no sean originales o que comprometan la autenticidad del conjunto, dando así al edificio una armonía compositiva para retomar el valor estético originario.

Sobre la base del análisis de los elementos característicos de la arquitectura local, pueden desarrollarse múltiples soluciones decorativas diferenciadas por períodos históricos, cuyas consideraciones permitirán intervenir de forma adecuada sobre lo construido. De este manera las intervenciones no serán genéricas, sino enfocadas y compatibles con las distintas tipologías de edificación. Cierra esta tercera fase, el proyecto de equipamiento de los espacios urbanos donde se elegirán los materiales y los modelos compositivos para las nuevas pavimentaciones, a través de las reflexiones surgidas del análisis del estado del conjunto artístico y de las aportaciones que se pudieran obtener de las fuentes históricas e iconográficas. La intervención en la iluminación pública, la señalización y el mobiliario urbano, serán fruto del compromiso entre el proyecto y las características peculiares de cada centro histórico objeto de estudio.

Lamentablemente, todavía hoy en muchos centros históricos italianos se asiste a intervenciones de sustitución edificatoria, con demoliciones de edificios de interés. En la Via Proconsolo de Florencia, las demoliciones han sacado a la luz restos de muros y de una cisterna romana. En el espacio comprendido entre la Badia y el Palazzo Bargello, aparecieron fragmentos de muros y una torre circular, que se significaron en el pavimento, mediante un listón de bronce.
Más allá de los descubrimientos arqueológicos, los nuevos edificios no tienen en cuenta, por lo general, ni el contexto en que se encuentra ni otro tipo de indicaciones relativas a la inserción de arquitectura moderna en cascos históricos.

Otros ejemplos a tener en cuenta en Florencia, podrían ser el edificio que se encuentra frente a los Uffizi, en la Piazza del Grano, un inmueble con sus voladizos y revestimiento en piedra, que retoma las líneas de la arquitectura del renacimiento, pero que no llega a integrarse en el entorno. Igualmente la intervención de restauración del conjunto de las Murate, donde se han puesto de relieve los elementos antiguos introduciendo elementos modernos, como los bow windows y los balcones apoyados sobre elementos verticales, si bien en este caso el conjunto resulta armonioso y funcional (Figuras 10, 11 y 12).

En la actualidad, cada vez se considera más importante reiterar que el tejido urbano se conserve en su complejidad, y no solo distinguiendo entre edificios con más o menos mérito. Las intervenciones que se desarroIlen en estos ámbitos deben ciertamente permitir nuevos usos, pero manteniendo la ciudad con la imagen estratificada que el tiempo le ha dado.

En Italia, el D. M. del 2008 sobre las construcciones en fábrica tradicional, ha añadido nuevos parámetros para la intervención sobre los edificios históricos. El nivel de conocimiento, que permitirá la fiabilidad en cada edificio antes de intervenir, obliga a llevar a cabo una serie de investigaciones preliminares, tales como el levantamiento arquitectónico y estructural, el estudio de las fases evolutivas y una atenta mirada a su evolución histórica. Sólo completando este itinerario, con la documentación requerida, se podrá intervenir con factores de riesgo reducidos al mínimo. Si no se conoce bien el elemento sobre el que se pretende actuar, cualquier intervención será genérica y no enfocada a resolver el problema del edificio o elementos concretos del mismo,
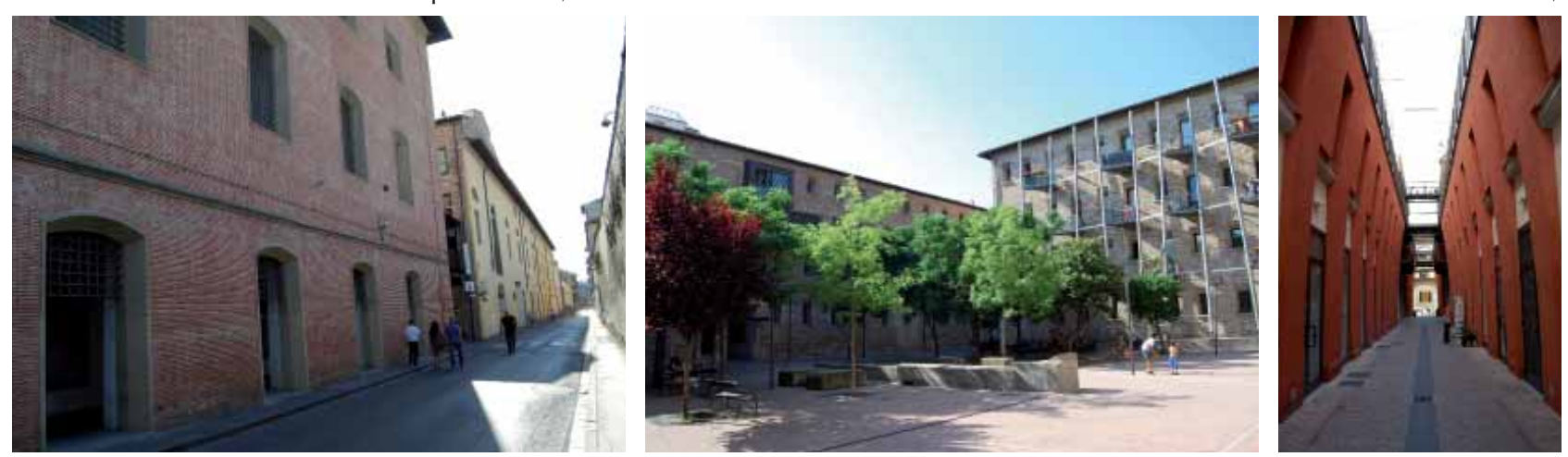

10

11 
como se ha podido ver después de recientes catástrofes y seísmos.

Una mala restauración estructural, puede ser más peligrosa que la falta de intervención. Ejemplos evidentes han sido el hundimiento de las bóvedas de la Basílica Superior de Asís, probablemente debido a la realización de un zuncho perimetral que rigidizaba la misma. Igualmente el hundimiento de la cubierta de la catedral de Noto. En esta, se creía que los pilares eran macizos, construidos en piedra de cantería, pero en realidad sólo la parte epidérmica del pilar, su perímetro, estaba formado por piedra maciza, mientras que su interior estaba constituido por un vertido a sacco, y con el paso de los años, la entrada de agua hizo colapsar los pilares provocando el hundimiento.

Todos estos problemas surgen generalmente de un conocimiento superficial del edificio, a menudo limitado a una pequeña búsqueda bibliográfica, frecuentemente de índole muy general, un levantamiento no muy riguroso, sin la ayuda de la instrumentación necesaria para realizar las debidas comprobaciones, una falta de conocimiento de sus técnicas constructivas y una separación, entre las intervenciones de restauración y las de consolidación.

Generalmente, no se tiene en cuenta que el término "consolidación" nace de la evolución de la restauración estática que, como ciencia, pertenece más al campo de los arquitectos que al de los ingenieros, si bien en la actualidad se actúa mediante equipos interdisciplinares, dirigidos normalmente por un arquitecto.

El problema del análisis estructural, es un punto importantísimo de la didáctica en la Escuela de Florencia desde hace varias décadas, estando orientada al conocimiento del modelo estructural y a las verificaciones estáticas y sísmicas que le están vinculadas. El profesor Piero Sanpaolesi, fundador de la escuela de Florencia, consiguió transformar la práctica de la restauración, de históricoartística a histórico-científica; Sanpaolesi pensaba que "un edificio estará tanto mejor restaurado, cuanto más parecido se comporte estructuralmente a su comportamiento inicial", demostrando que la Universidad en sus investigaciones, se anticipa al legislador. La Cátedra de consolidación y adaptación de los edificios se activó en toda la universidad de Florencia a finales de los años cincuenta y ha visto en sus aulas a los profesores Sisto Mastrodicasa, Lorenzo Nizzi Grifi y Silvio Van Riel.

Aunque han avanzado mucho las nuevas tecnologías, que sirven de ayuda a los téc- nicos especialmente en la fase de toma de datos, sigue siendo indispensable el contacto directo con el edificio de estudio, pues se correría el riesgo de conocer bien la geometría del edificio, sin conocer realmente su naturaleza.

\section{DOS INTERVENCIONES ACTUALES EN FLORENCIA}

En esta visión de la protección y salvaguarda de los edificios históricos, podemos contemplar dos intervenciones actualmente en fase de realización en Florencia.

El primero es del Palazzo de Giuliano Dami, protegido del gran duque Giangastone de Medici, donde intervinieron los más importantes artistas y arquitectos de la primera mitad del siglo XVIII, como Ferdinando Ruggeri, Giovacchino Fortini y Niccolò Pintucci.

El edificio, objeto de una importante intervención de adecuación en el siglo XIX y además siendo en su origen utilizado como residencia de un único propietario, fue transformado en apartamentos individuales. Además, en este proyecto se intervino en elementos singulares de importancia, tales como la chimenea del comedor, en la del salón y en las dos fuentes. Sus realizadores quisieron que el proyecto fuera de protección del inmueble, pero también de modernización y organización funcional.

La planta noble del Palazzo Dami, se caracteriza por un importante salón que se asoma sobre la Via Maggio, salón al que se opone simétricamente la habitación principal del propietario, que se abre a la vía Borgo Tegolaio. Entre estos dos ambientes se desarrolla el Palazzo, sobre un eje de unos 30m.

Conecta todos los ambientes la galería pintada en el settecento por Pintucci, en que los fragmentos de ruinas sirven en primer plano de fondo a la arquitectura ideal. En la parte central, frontalmente a las tres únicas ventanas del vano, se abre una gran terraza en perspectiva, dibujada con numerosos puntos de fuga.

Se abren a la galería el estudio, la sala comedor, y un ambiente que probablemente servía como antesala de la cámara del duque; lateralmente, los ambientes de servicio se desarrollan adosándose al edificio vecino.

El proyecto de restauración, ha afectado a la totalidad de la planta noble, y también a un entresuelo, que antiguamente estaba destinado a cocina, y a las bodegas, todos ellos ambientes conectados por una barroca escalera de caracol. 
13. Florencia, palazzo Dami, galería de la Planta Noble, en fase de restauración.

14. Florencia, palazzo Dami, galería de la Planta Noble, después de la restauración.

15. Florencia, palazzo Dami, viga de acero en la cual descansa la instalación completa de climatización al lado de la cocina antes de terminar el trabajo.

16. Florencia, palazzo Dami, la pared descansa en la parte frontal de la capilla hasta que el trabajo esté terminado.
Los ensayos realizados en los revestimientos, efectuados en la fase preliminar, han sacado a la luz los frescos de la galería, que se habían ocultado probablemente en el siglo XIX. Lamentablemente, han sido las únicas pinturas murales restauradas, aunque otras pinturas también fueron detectadas mediante los ensayos efectuados sobre el artesonado del salón principal, decorado con rombos y palmeras sobre fondo rojo, decoración común de fin cinquecento o comienzos de la siguiente centuria y consiguientemente del Barroco.

El descubrimiento de estos elementos decorativos, sirvió de guía al proyecto de restauración, dando al apartamento un aire barroco, manierista, propio del mundo florentino, como si fuera ideado por el cliente original.

Después de la intervención de limpieza de la decoración de la galería, se procedió a realizar una campaña fotográfica con equipos especiales, que ha permitido reconstruir fotogrametrías fiables de las fachadas, a pesar de su forma oblonga (Figuras 13 y 14).
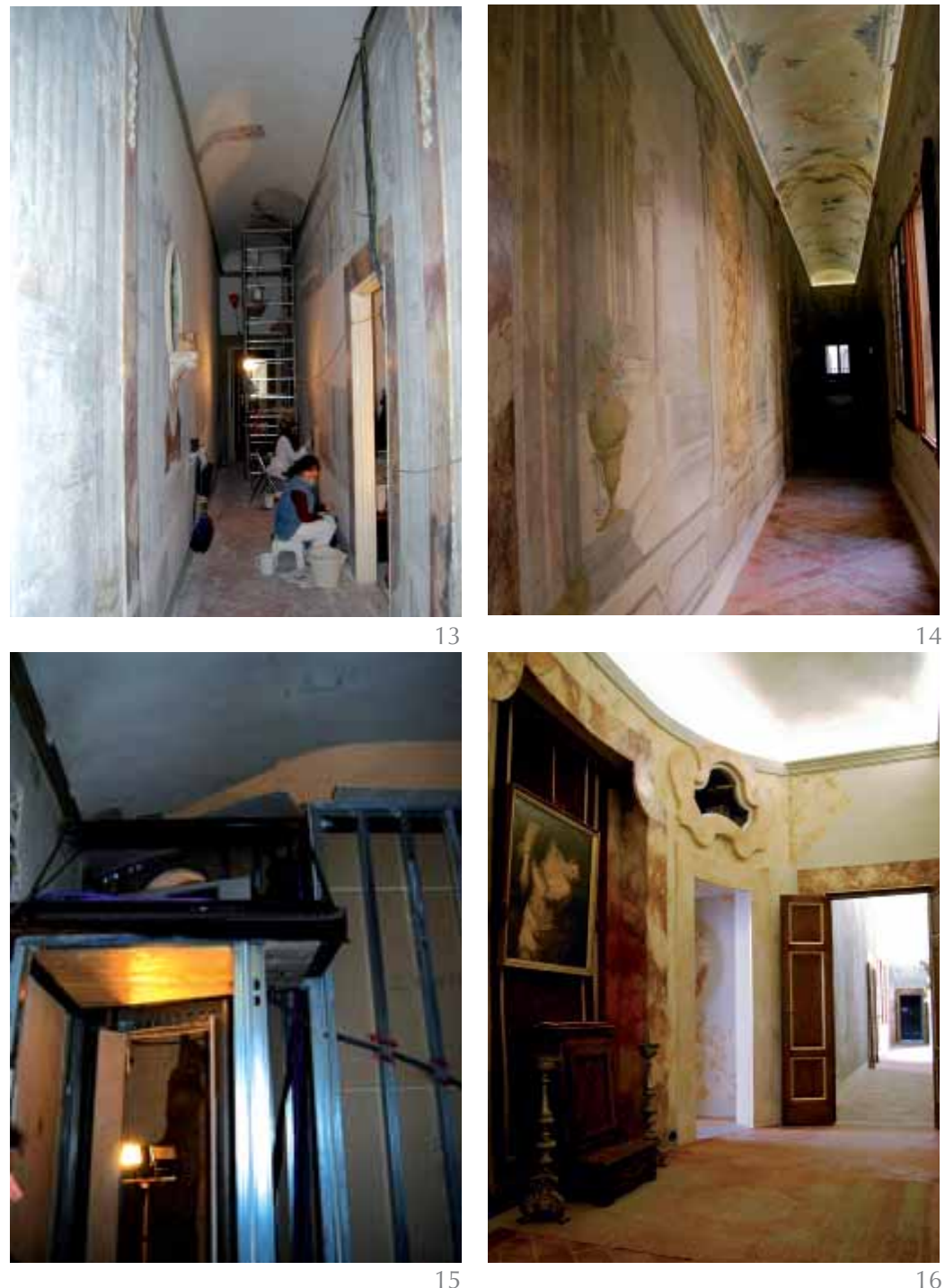

Sobre la base de estas fotogrametrías, se ha procedido a identificar los puntos de fuga, la línea de tierra y los planos prospectivos, lo que ha permitido intervenir en las integraciones, sin modificar la geometría de los entornos, sobre todo en la parte central donde la decoración es poli focal. Se ha mantenido ese sentido de movimiento que el visitante tiene cuando atraviesa la galería, pues si en la decoración con punto de vista central la pintura es observada desde un único punto, (porque más allá de aquel aparecerá deformado), en aquellas poli focales, sin embargo, quien recorre el espacio, tiene constantemente la sensación de que la perspectiva se mueve con él hasta el final del recorrido.

Para la reintegración pictórica, se ha decidido intervenir unificando la imagen y permitiendo sólo a quien se aproxima a la pintura leer la restauración. Sólo en dos casos han sido realizados elementos nuevos, uno es el del dintel de la entrada principal que no existía, puesto que realizada en el ottocento y el otro, constituido por las dos ménsulas próximas a la escalera.

Estas intervenciones se consideraron necesarias, en cuanto la galería discurría hasta el salón, y con la división en habitaciones, esta había sido interrumpida y se había convertido en lugar de desembarco de las escaleras de acceso a los ambientes de la planta de arriba. Dentro del apartamento, esta ruptura creaba una sensación de algo inacabado, incompleto, que se decidió solucionar "cerrando" pictóricamente el ambiente, utilizando elementos ya existentes en el repertorio decorativo de la galería. La intervención ha sido firmada y fechada para permitir su inmediato reconocimiento.

El espacio correspondiente a la cabecera de la galería, aquel que probablemente fuera la antesala de la cámara de Giuliano Dami, fue dividido en tres ambientes, para permitir la realización de la cocina, que antiguamente estaba en el semisótano.

El levantamiento estructural, puso en evidencia los problemas de la solería de madera. Para proceder a su consolidación, se comenzó dividiendo la solería en cuatro partes, y a intervenir por cuadrantes alternos, sin descargarla nunca completamente.

El delicado equilibrio estructural de esta planta, ha exigido que todos los elementos pesados no cargasen directamente sobre los suelos. De hecho, se ha colocado una viga de acero, apoyada sobre los dos muros de carga, que sostiene las instalaciones de climatización de esta zona del edificio, y una gran librería, existente en el centro de la habitación (Figuras 15 y 16). 
La librería, se apoya igualmente en la viga, no grava sobre el pavimento y es llevada a tierra por medio de dos pies regulables, para evitar las oscilaciones. Todo esto ha quedado oculto por elementos reversibles, decorados para dar continuidad a la galería.

Cierra la crujía la habitación del propietario, donde la limpieza superficial ha sacado a la luz unas pinturas de principios del siglo XX, que se ocultaron para dar al apartamento la unidad estilística imaginada por el cliente. En este caso, la consolidación del forjado, se ha efectuado mediante red electro soldada, con entrega en los muros perimetrales y unida mediante conectores a las viguetas subyacentes; de este modo se obtuvo un plano rígido de enlace entre los muros (Figuras 17 y 18).
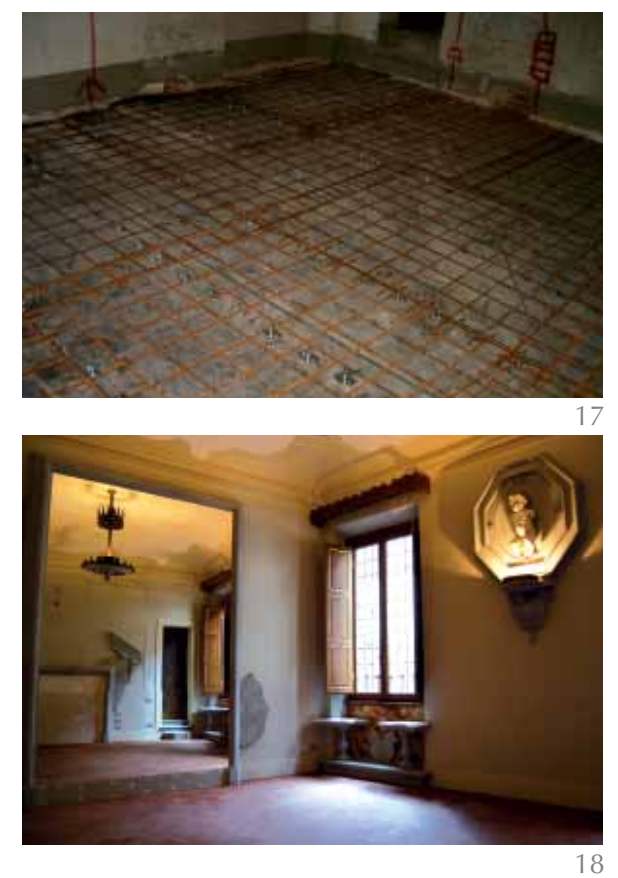

El forjado contiguo que sostiene los baños y el guardarropas, está constituido por viguetas de hierro y aligeramientos cerámicos; también en este caso se extendieron mallas electro soldadas, ancladas a las paredes, y como conectores se soldaron a las viguetas de sección en IP, luego soldadas a la malla; también esta intervención convirtió en rígido y solidario el forjado de esta zona de servicios (Figuras 19 y 20).

Todos los elementos de ornamentación en piedra, fueron simplemente atornillados a las paredes, para permitir un eventual desmontado sin crear problemas a las estructuras $^{3}$, en la perspectiva de una perfecta reversibilidad de las intervenciones. La utilización de elementos característicos de la cultura toscana, como el cocciopesto para los pavimentos de algunos ambientes, la pietra serena para los elementos decorativos, el pórfido rojo, de memoria romana, para los lavabos, contribuyeron a crear un ambiente bien definido.

La segunda obra analizada es la correspondiente a los Ilamados "Nuevos Uffizi", efectuada en uno de los más importantes monumentos italianos, conocido también por la colección de obras de arte que alberga. El edificio, proyectado por Giorgio Vasari, por encargo de Cosimo I para albergar las colecciones magistrales de los Medici en una zona de la ciudad fuertemente urbanizada.

Para la construcción del complejo de Vasari, fueron demolidos los edificios que ocupaban la zona, salvo las estructuras de la iglesia de San Piero Scheraggio y de la torre de la vieja Zecca, que fueron incorporadas ${ }^{4}$.

En la última década, los Uffizi, tras la liberación de toda la primera planta y de una buena parte de la planta baja, ya ocupada por el Archivo de Estado, han sido objeto de importantes obras de restauración, que no sólo intentan facilitar la visita al museo, sino también hacer visibles elementos ocultos, así como las colecciones conservadas en los depósitos.

El nuevo destino de los espacios, ha hecho necesaria la consolidación de la fábrica de Vasari. Han sido proyectadas algunas intervenciones estructurales, con objeto de eliminar los daños debidos a la utilización del inmueble por parte de la administración anterior, así como la abertura de puertas a varios niveles. Ha sido también necesario realizar obras de musealización, en espacios que habían sido creados con otros objetivos.

El proyecto, supervisado directamente por la Soprintendenza per i Beni Architettonici, Paesaggistici e Etnoantropologici de las provincias de Florencia, Pistoia y Prato, y específicamente por la responsable del control arquitecto soprintendente Alessandra Marino ${ }^{5}$ y la arquitecto Laura Baldini como directora de las obras ${ }^{6}$, ha sido elaborado por el estudio S.in.terSrl, de Florencia (Ing. Alessandro Chimenti y arch. Alessandro Moroni) con el asesoramiento de los arquitectos Adolfo Natalini y Piero Castiglioni.

La dificultad de intervenir en una fábrica tan compleja, en que los problemas de restauración de conservación se enfrentan a menudo con las necesidades de consolidación, musealización y disfrute de los lugares, ha exigido a la soprintendenza un gran esfuerzo de energías y capacidades técnicas y organizativas, en unas obras que no contemplan el cierre del recorrido principal del museo.

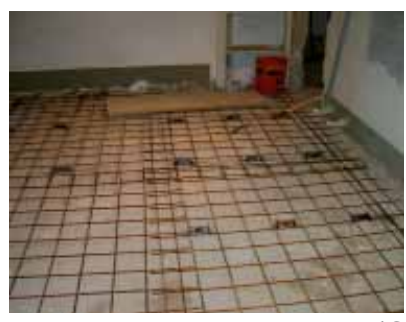

19

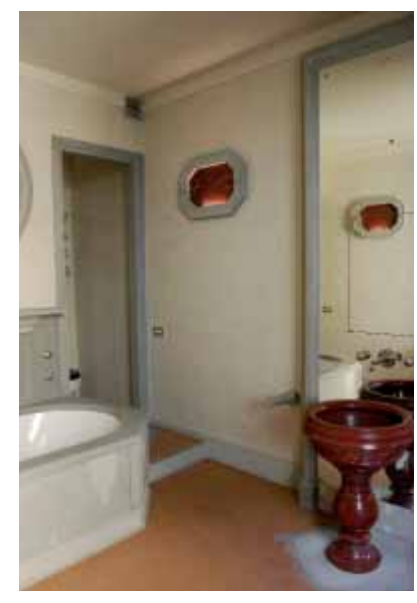

20

17. Florencia, palazzo Dami, habitación principal durante la intervención de consolidación del forjado.

18. Florencia, palazzo Dami, habitación del propietario con la obra terminada.

19. Florencia, palazzo Dami, cuarto de baño durante la intervención de consolidación del forjado.

20. Florencia, palazzo Dami, cuarto de baño con la obra terminada.

${ }^{3}$ La intervención ideada por el propietario, el escultor Federico Mario Caruso, ha visto como director de trabajos al que escribe desde el 9-112009 y realizador de la modificación en fase de obra desde el 18-6-2010.

${ }^{4}$ Las estructuras fueron encontradas durante los actuales trabajos

${ }^{5}$ Responsable único del proceso, que ha sucedido a la arquitecto Paola Grifoni, hoy soprintendente en Bologna, que ha seguido la obra de 2006 al 2009.

${ }^{6}$ Que ha sucedido al arquitecto Papagayo, que siguió las obras del 2006 al 2010 . 
21. Florencia, Piazzale degli Uffizi. 22. Florencia, Piazzale degli Uffizi, construcción vertical con 9 niveles.
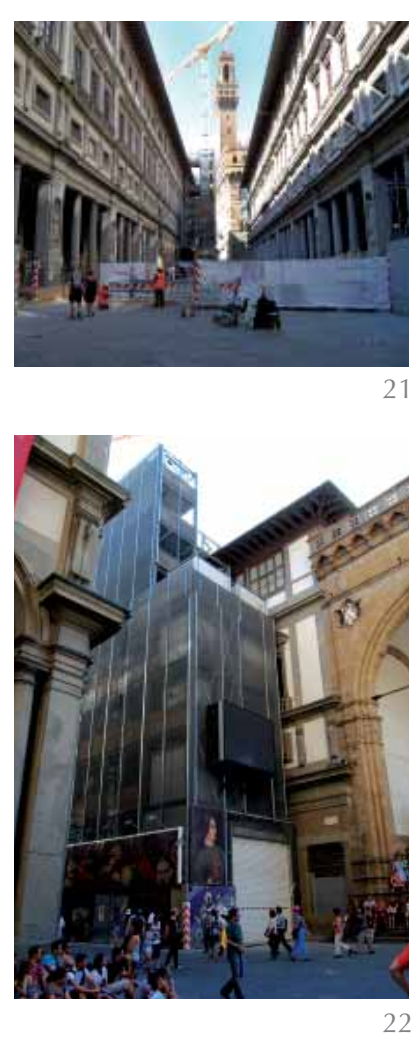

22
El proyecto, incluye también la realización de elementos arquitectónicos completamente nuevos: además de la salida proyectada por Arata Isozaki, objeto de un importante debate nacional, están previstas dos escaleras de enlace y salida, diseñadas por Adolfo Natalini y perfectamente insertadas en el complejo histórico de Vasari.

El primer problema que hubo que afrontar fue el de la organización de las obras, puesto que la forma misma del edificio no favorecía la optimización de las mismas. Se diferenciaron cinco áreas distintas, con abastecimiento de materiales anejos, y se hizo necesario organizar en la plaza de los Uffizi la llamada "máquina de las obras". Es decir, un elemento vertical con 9 niveles y grúa incluida, que acoge en su interior los vehículos y permite la descarga de los materiales de construcción en el interior, sin interferir con la visita turística. La realización de estas cinco áreas de obra, supuso un aumento de los costes de seguridad y de las propias obras.

La compleja intervención estructural ha sido dirigida por el Ing. Sergio Lagomarsino, asesor del Ministerio de Bienes y Actividades Culturales ${ }^{7}$.

Los planos horizontales fueron consolidados con losas armadas, pero sin zunchos de hormigón armado, si no utilizando un "cinturón" de acero, formado por perfiles en " $C$ " unidos con elementos atornillados pasantes; a estos, fueron soldadas barras horizontales para el anclaje de tirantes pla- nos, que unen también transversalmente el cinturón metálico, para limitar el espesor y no cambiar el nivel del suelo.

Es interesante también, el sistema de consolidación de los arcos que sostienen el suelo de la primera planta, y que cargan sobre las bóvedas de los espacios y de la loggia inferior. Para ello, se construyeron arcos de madera laminada con tres articulaciones (bisagras), que refuerzan la estructura sin hacerla rígida.

Los cercos para la abertura de nuevos huecos, se realizaron respetando lo más posible la estructura de los muros: con perfiles metálicos en "L" en lugar de en " $\mathrm{H}$ " para acoplarse al espesor, sin hacer necesarias grandes aberturas en los muros, mixtos en la mayor parte de los casos y por tanto de difícil delimitación.

La problemática del paso de las instalaciones ha sido uno de los más difíciles de resolver, por la necesidad de actuar sobre las fábricas, con el máximo respeto al equilibrio estático de las estructuras. Por ejemplo, "encamisando" los orificios pasantes, para evitar la pérdida de resistencia del conglomerado de los muros en los puntos afectados.

En conclusión, la delicadeza del contexto en el que se actúa, ha hecho necesario en esta obra, prestar una especial atención a los nuevos métodos empleados, para actuar de la forma más armónica con el elemento arquitectónico y para ser más respetuoso con sus peculiaridades.

\section{BIBLIOGRAFÍA}

(1) Romeo,E.: Verso una storia del restauro, pp.13-15, Alinea editrice. Firenze, 2008.

(2) Rocchi Coopmans de Yoldi, G.: S.Maria del Fiore. Teoria e storie dellárcheologia e del restauro nella cittá delle fabriche arnolfiane. pp.396-398, Alinea editrice. Firenze, 2006.

(3) Gurrieri, F. I.; Sembrini, P.; Van Riel, S.: Il restauro del paesaggio, Alinea editrice. Firenze, 2005.

(4) Roselli, P.: "Restaurare la cittá, oggi". Storia e Restauro n 7, pp.11, 1991. 